\title{
Cluster Parallelism of the Neuroprocessor Systems Based on the Fuzzy Logic
}

\author{
Ruchkin Vladimir Nicolaevich ${ }^{1}$, Kostrov Boris Vasilyevich ${ }^{2}$, Romanchuk Vitaliy \\ Alexandrovich ${ }^{1}$, Fulin Vladimir Andreevich ${ }^{1}$, Ruchkina Ekaterina \\ Vladimirovna $^{2}$ \\ ${ }^{1}$ Ryazan State University \\ ${ }^{2}$ Ryazan State Radio Engineering University
}

Keywords: neurorpocessor system, equivalence classes, clustering, fuzzy clustering, explicit and implicit parallelism, linguistic variables, intellectual compiler, fuzzy logic

\begin{abstract}
The article is devoted to the development of the neuroprocessor system (NPS) model based on the clustering and the analysis of the obtained fuzzy structures. The production model of explicit and implicit parallelism is suggested. The given model could be used for the intelligent compiler development and for the choice of the optimal technical parameters, such as NPS modules number, the firmware memory volume, the system productivity etc.
\end{abstract}

\section{Introduction}

The sequential nature of the computational process based on the J. Von Neumann's principles [1] shows the low productivity of the system in the whole. The vast majority of the memory cells are inactive during the process. So, the resource usage of the hardware is quite low [4]. Besides, the developer is forced to adapt the decision algorithm to the hardware structures available.

On the other side, the engineering realization of the theoretical neurons developed by W. McCulloch and W. Pitts [2] represented in the form of the neuroprocessors allows to get rid of the drawbacks mentioned above. Besides, with the using of the neuroprocessor system (NPS) [5] the developer can easily adapt it to the nature of the task being solved.

The cluster systems development brought to life several problems, such as a problem of the effective cluster partitioning $[8,10]$. Another problem is that of the more profound explicit and implicit parallelism analysis for the development of the high-productive parallel hardware using the "intelligent" compilation elements like VLIW, EPIC, SIMD etc. architectures.

The goal of the work is cluster neuroprocessor structures development for the creation of the new class of the intellectual systems using explicit and implicit parallelism [7] with "intelligent" compilation elements.

The main task is to develop the designing procedure for the cluster NPSs and the explicit and implicit parallelism analysis of the structures obtained, which can be implemented according to the different technical parameters, such as performance, the firmware memory volume and NPS modules number.

\section{The explicit parallelism model of the NPS choice system production}

The suggested model of choice is based on the functional principle. According to it, the set of operations is considered the main criteria [3].

On the first stage, according to the functional principle, the NPS is seen as a totality of the hardware embodied on the base of the chosen hardware base.

Thus here is the choice of the hardware base with the set of commands, productivity and value [9]. The projectable computational structure of the NPS is functioning according to the data processing and data transfer algorithms $A^{(j)}, \forall j=\overline{1, N}$, Each of them is represented by a tuple of operations $A^{(j)}=<O_{1}, O_{q}, \ldots, O_{L}, O_{2}, O_{1} \ldots, O_{r}>$ with the length of $\left|A^{(j)}\right|$ or the algorithms of contraction, 
filtering cull etc.

The mathematical solution is represented in the finding of the representation

$$
O_{I} \rightarrow\left\{M K_{m}\right\} ; \forall m=\overline{1, K_{m}} ; \forall l=\overline{1, L},
$$

where the multitude of the commands of the specified set of the integrated circuit $M K=\left\{M K_{1}, M K_{2}, \ldots, M K_{m}\right\}$ is considered as the inner computing system (CS) language. $M K_{m}-$ is the minimal number of commands performing an operation $O_{I}$. The system of the commands applied in NM 640X is used here as an example.

The second stage depends on the projecting task (1) solution. Here the correspondence between each ${ }^{j}$-th processing algorithm and the processing software $P R^{(j)}$ is found. It is done with the determinate representation:

$$
A^{(j)} \rightarrow P R^{(j)}, j=\overline{1, N}
$$

The data processing software $P R^{(j)}$ is represented as a tuple of commands: $P R^{(j)}=<M K_{1}, M K_{2}, \ldots, M K_{i}, \ldots, M K_{M}>$.

The main characteristics of the software under cincern $P R^{(j)}$ are: the software length $\left|P R^{(j)}\right|$, defined as a general number of the commands compiling the; software run time $T=\sum_{m-1} t_{m}$, where $t_{m}$ - the run time of the $m$-th command. The B expression (2) in the general case defines the compilation process of the single-procession version of the NPS. And it corresponds to the centralized data processing structure.

To solve the task of the partition $P R^{(j)}$ the authors introduce a conception of the equality of the $\mathrm{CL}_{\mathrm{l}}$ и $\mathrm{CL}_{\mathrm{k}}$ clusters of the some processing software $\mathrm{PR}^{(\mathrm{j})}: \mathrm{CL}_{\mathrm{l}}=\mathrm{CL}_{\mathrm{k}}$, Further the equality will be considered as an equality of the lengths of the specified clusters $\mathrm{CL}_{\mathrm{l}}=\mathrm{CL}_{\mathrm{k}}$ and their coincidence to within the command $\mathrm{Mk}_{\mathbf{i}}^{(\mathbf{k})}=\mathrm{MK}_{\mathbf{i}}^{(\mathbf{l})}, \mathrm{PR}^{(\mathrm{j})}=\left\langle\mathrm{MK}_{\mathrm{i}}, \mathrm{MK}_{1}, \ldots \mathrm{MK}_{\mathrm{j}}\right\rangle$, so that.

$$
C L_{l}=C L_{k}=\left\{\begin{array}{l}
\left|C L_{l}\right|=\left|C L_{k}\right|, \forall l, k=\overline{1, N} \\
M K_{i}^{(l)}=M K_{i}^{(k)}, \forall i=\overline{1,\left|C L_{l}\right|}
\end{array}\right.
$$

To define and to introduce the clusters the authors suggest using the theoretic-set model based on the conception of equivalence [4].

Further the concept of the cluster structure $S_{w} \in S$ is introduced. The cluster structure is a ratio between the running sub-programs $R Q, R Q_{k}$ concurrency in the form of the clusters $\mathrm{CL}_{\mathbf{l}}$ и $\mathrm{Cl}_{\mathbf{k}}$ [6] by two different neuroprocessors or modules: $C L_{l} S_{w} C L_{k} ; C L_{l}, C L_{k} \in P R$ of the projectable NPS.

Then on the third construction stage the multitude of the various cluster structures $S_{w} \in S$, is defined. These structure allow some data ${ }^{j}$-th processing software $P R$ to put the multitude of the clusters into the coincidence $\mathrm{CL}_{\mathrm{l}} S_{w} \in S: P R \Rightarrow\left\{C L_{i}\right\} ; \forall i=\overline{1, l} ; \forall w=\overline{1, W}$

The expression (4) defines the explicit parallelism analysis and is corresponding to the distributed structure $S_{w} \in S$ of the NPS.

The fourth stage is connected with the optimal choice of the data processing NPS structure according to the given construction strategy. In includes the minimum of the hardware and software and is satisfactory to the production requirements.

Thus, the data processing NPS structure $S_{w}$ puts into the correspondence some $j$-th data processing software $P R$ and the totality of the independent and unequal clusters $C_{\mathrm{l}}$, the number of which is is equal to the number of the equivalence classes $L$, and ratio ( $\left.R O_{l}\right)$ is defined by the order of the equivalence class order $\left(a_{l}\right)$ : 


$$
\forall o=\overline{1, N}, P R^{(j)} \Rightarrow\left\{\left(R O_{l}\right) q\right\}, \forall q=1, a_{l} ; \forall l=1, L
$$

Further the authors look through the following alternatives of the task solution (5) in the form of the Expert system production model.

\section{The Production Model of the Expert System for the NPS Choice}

In the task (5) solving process there are the following variants of the NPS structure organization.

1. The equivalence classes number is equal to $L$, and the order of each $l$-th class is equal to $\left|a_{l}\right|$. Then $i_{\text {-th }}$ data processing NPS algorithm is put into the correspondence with the data totality which idls defined according to (5)/ So, this case is the most general and it characterizes the multineuroprocessor structure like MKMD (according to Flinn), because it presupposes not only the relations between the elements inside one class like in the matrix structure, but also the relations between the elements of different classes like in the pipeline structure.

The alternative structures of the data processing NPS considered below are possible depending on the build of the specified $j$-th data structure $P R^{(j)}$, but, more precisely, on the data transfer between these structures.

Let's assume that the data exchange between the representative of the $C L_{l}, l=\overline{1, L}$ clusters, is performed sequentially from one to another.

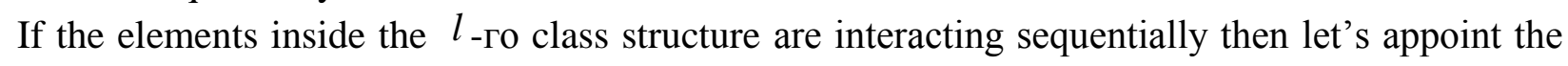
number of the fuzzy sets (FS) for the case $\left|a_{1}\right|=q, \forall l=\overline{1, L}$, equal to $N_{0}=L \cdot q$, and for the general case $N_{0}=L \sum_{l=1}^{L}\left|a_{l}\right|$. As a result, the already known pipeline structure is obtained. And the hardware and software and temporary characteristics for the system sre defined by the following correlations.

The obtained system with the number of the computing facilities $N_{\mathrm{O}}=L \cdot q$ is functioning according to the clusters for the case $\left|a_{1}\right|=q, \forall l=\overline{1, L}: C L=q \sum_{l=1}^{L} C L_{l}$ and for general case $C L=\sum_{l=1}^{L} \sum_{m=1}^{M} C L_{l m}$. The data processing time is calculated on the formulae: $T_{r}=q \sum_{r \in R O_{l}} t_{i}^{(1)}$, $T_{r}=\sum_{l=1}^{L}\left|a_{l}\right| \sum_{r \in R O_{l}} t^{(l)_{i}}$ correspondingly for the first and for the second case.

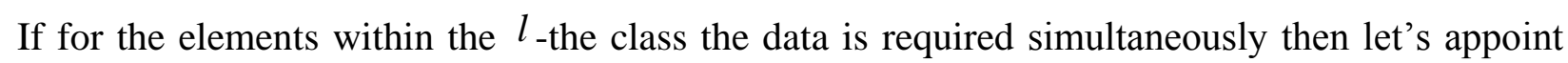
the number of the NCS modules for the case $\left|a_{1}\right|=q, \forall l=\overline{1, L}$, equal to $N_{0}=L \cdot q$, and for the general case $N_{0}=L \cdot \max _{l \in L}\left|a_{l}\right|$. The obtained structure is called the vector-pipeline NCS structure. If the data is required simultaneously for all the users during the processing, then then the number $N_{0}$ of the processor modules is appointed to be $N_{\mathrm{o}}=L \cdot q \quad \forall l=\overline{1, L} ;\left|a_{l}\right|=q$ и $N_{0}=\sum_{l=1}^{L}\left|a_{l}\right|$ for the general case. As a result, the matrix neurocomputer structure of the data processing is obtained.

In the most general case during the input data processing the input data can be required by some neurorpocessors simultaneously or be transferred sequentially between a number of the rest. In other words, the line of the tasks is parallelized like in the vector structure, and the other line of the tasks is parallelized like in the pipeline. As a result, the complicated pipeline-vector or vectorpipeline computer system structure. The number of the neuroprocessors in such a structure in the system is defined according to the known correlation. Nevertheless, two more aspects should be mentioned. First, the pipeline, pipeline-vector, vector, vector-pipeline and in matrix structure in the general forms are considered as a multitude of the various configurations. Each of them should also 
be classified, e.g. with the using of the linguistic variables based on the fuzzy sets principle [11]. Second, the complicated multineuroprocessor systems can be implemented with the help of the crystal based on the multinuclear processors. The given correlations can be considered the basic for the expert system learning.

\section{The Implicit Parallelism Analysis by Means of Multicriterial Fuzzy Choice}

In practice, the NPS constructing process is a complicated multicriterial task of the choice of the structure from the obtained variety $S_{w} \in S$. The decision-maker should make choice of the best alternative on the base of the estimation of each one. So, this person makes a decision guided by the totality of the discrepant criteria: speed $\left(1 / T_{R}\right)$, the number of the firmware memory volume $(|M P|)$, the microprocessor modules $\left(N_{0}\right)$, the downtime $(\operatorname{Tn} p)$ etc. To specify the choice it is suggested to rate all the listed above criteria according to the strategic construction importance. The process is suggested to be called the construction strategy.

The suggested multicriterial choice methodology is based on the fuzzy sets. Within the fuzzy sets the characteristic function has a segment $E=[a, b]$ as a definitional domain and the linguistic variables are its elements.

The introduction of fuzziness allows to model the smooth change of the multineuroprocessor realization properties according to the qualitative structure connections. This factor is the most important for the neurolike webs. And the solving of the task of choice is conducted simultaneously with the decision-making procedure [12].

The decision-maker estimates the structures and the criteria using the concepts of the fuzzy variables or terms $t \in T$. And each variable has a definitional domain in the form of the segment $E=[a, b]$ and a membership function $m_{t}(e), \operatorname{so} t-\left\{m_{t}(e), e\right\}$.

The suggested methodology is based on the two kinds of the fuzzy variables. The first is a binary linguistic variable called "the criteria significance ratio" $R_{j \mid j+1}$ or "the structures utility ratio" $Q_{j \mid j+1}$. The second is the unary linguistic variable called "the qualitative estimation of the criterion" $m_{z j}(x)$ or "the qualitative structure utility estimation" $m_{i, j, s i}(x, y)$.

The value of the binary linguistic variables of "the criteria significance ratio" and "the structures utility ratio" are built by using a fuzzy algorithm method. The algorithm presupposes the rules of the "If $A$ than $B$ " from. Two and more rules of the fuzzy statements are united with the conjunction "else", e.g. "If $A$ than $B$ else $C "=[A][B]+[A]^{*}[C]$ or $(«$ If $A$ than $B$ else $C »)=$ $m_{A} / \bigcup(B), 1-m_{A} / \bigcap(C)$.

The significance estimation of each previous criterion in the list is carried out according to the construction strategy and with the implementation of the composition rule.

$Z_{j}=Z_{j+1} \circ R_{j \mid j+1}, j=n-1, n-2, \ldots, 1$. And the fuzzy set membership function $Z_{j}$ is a result of the maxmin operation:

$m_{z j}(x)=\bigcup_{y \in Y} m_{z j+1}(y) \bigcap m_{R j \mid j+1}(y, x)$.

To obtain the weighs of the significance criteria the fuzzy sets are transformed into scalars. It is done using one of the known methods, e.g. choosing of the binary variable value with which the membership function reaches its maximum. Then the scalar estimations of the criteria significance are scaled and normalized. As a result, each of the criteria gains weight $W_{i}, \forall l=\overline{1, n} ; \sum_{l=1}^{n} W_{l}=1$.

According to the described principle the normalized utility evaluation on each $j$-th criterion $F_{i j}$. is calculated from the linguistic estimates of the utility ratio. 
The integral characteristic of the multicriterial fuzzy choice suggested is the additive utility function $U_{i}$, calculated in the final part of the methodology described for the alternatives of the structure $S_{w}$ of the data processing according to the totality of all the criterions. Besides, the obtained variants are rated according to the volume $U_{i}=\sum_{j=1}^{n} W_{j} * F_{i j}$.

The preferable alternative of the structure $S_{w} \in S$ NPS with the maximum utility function is defined in correspondence to the obtained data.

As a result, the inferencing algorithm including the following steps was created:

1. The carrier choice and the obtaining of the initial distribution.

2. The constructing strategy definition through the rating of the specified criteria.

3. The definition of the value of the binary linguistic variables as «the criteria significance ratio» $R_{j \mid j+1}$, which is calculated according to the composition rule $Z_{j}=Z_{j+1} \circ R_{j \mid j+1}$, with the criterion choice beginning from the last one $j=n-1, n-2, \ldots, 1$.

4. The acquisition of the "the qualitative structure utility estimation" using the formula $m_{z j}(x)=\bigcup_{y \in Y} m_{z j+1}(y) \bigcap m_{R j \mid j+1}(y, x)$.

5. The acquisition of the scalar estimations $X_{j}^{k}$, thus the definition of the number of the carriers at which the criteria volumes reach 1.

6. The estimation scaling on the formula $H_{i}=3\left(X_{j}^{k}+1\right)+1$.

7. The normalized weighs acquisition on the formula: $W_{j}=H_{j} / \sum_{j=1}^{n} H_{j}$.

8. The definition of the binary linguistic variables volumes "the structures utility ratio" on the formula: $Z_{j}=Z_{j+1} \circ R_{j \mid j+1}$, with the choice of the structure beginning from the first.

9. The definition of "the qualitative structure utility estimation" value according to the formula $m_{z j}(x)=\bigcup_{y \in Y} m_{z j+1}(y) \bigcap m_{R j \mid j+1}(y, x)$.

10. The acquisition of the scalar estimations $Y_{i j}^{k}$, i. e. The definition of the number of the carriers at which the structure volumes reach 1 .

11. The estimation scaling on the formula $H_{i j}=3\left(Y_{i j}^{k}+1\right)+1$.

12. The normalized weighs acquisition $F_{i j}=H_{i j} / \sum_{j=1}^{n} H_{i j}$.

13. The calculations iteration from the p.8 for the whole set of the criterions.

14. The calculation of the additive utility function on the formula: $U_{i}=\sum_{j=1}^{n} W_{j} * F_{i j}$ alternative structures of the neurocomputer realization of the constructing neurocomputer on the all criteria totality according to the construction strategy.

15. The output of the logical solution neurocomputer construction result.

The information given above and the strategies formulated are the input data for the inferencing algorithm functioning. All the information obtained is put into a table. The table also contains the sections of the qualitative criteria estimation $Z_{j}=Z_{j+1} \circ R_{j \mid j+1}$, scalar estimations $X_{j}$, scaled estimatio $H_{i j}=3\left(Y_{i j}^{k}+1\right)+1$ and normalized weights $W_{j}$ for each criterion $W_{j}=H_{j} / \sum_{j=1}^{n} H_{j}, j=1, n$.

Further the binary linguistic relations of the utility alternatives are built on each criteria. Then the similar calculations of the $Y_{i j}$, scaled estimations $H_{i j}$, formalized weights $F_{i j}$, which are also included into the table. 
On the base of the obtained data the additive utility function $U_{i}$ is calculated of the alternative neuromicroprocessor structure variants of the neurocomputer realization on the totality of all given criteria: $U_{1}=\sum_{j=1}^{n} W_{j} * F_{i j}=0.3 ; U_{2}=\sum_{j=1}^{n} W_{j} * F_{2 j}=0.32 ; U_{3}=\sum_{j=1}^{n} W_{j} * F_{3 j}=0.38$.

\section{Conclusion}

The article is devoted to the generalized NPS construction model. The model is based on the clustering in the form of the equivalence classes. The expert system for the implicit parallelism analysis is suggested. The analysis is conducted by means of the multicriterial fuzzy choice. It is used for the intelligent compiler realization, carried out according to the different technical parameters. The compiler is developed taking into consideration different technical parameters and the construction strategy: speed $\left(1 / T_{R}\right)$, the firmware memory volume $(|M P|)$, the microprocessor modules number $\left(N_{0}\right)$. The suggested model is independent on the specific algorithms and doesn't define the tasks class. But the receiving of the specific NPS structure will depend on the algorithm and thus on the tasks class.

The results of the experimental research adduced in the Table 1, show the preferred variant of the vector-pipeline realization of the structure with the maximal utility function

This shows the similarity of the acquired results both on the commands and on the data.

\section{References}

[1] T.P. Baranovskaya, V.I. Loyko, M.I. Semenov, A.I. Trubilin, "The Computer Systems and Web Architecture", Moscow, "Finance and Statistics", 2003, 256 p.

[2] V.P. Koryachko, S.V. Skvortsov, T.A. Telkov, "Multiprocessor Systems and Parallel Calculations", Moscow, "Vishaya Shkola", 1999, 281 p.

[3] V.P. Koryachko, S.V. Skvortsov, I.A. Taganov, A.P.Shibanov, "The Evolution of the Automated Construction of the Elecronic Computers”, “The Radiotachnics”, 2012, № 3, pp. 97-103.

[4] R. Jordans, L. Jozwiak, H Corporaal., "Instruction Set Architecure Exploration of VLIW ASIPs Using a Genetic Algorithm", $3^{\text {rd }}$ Mediterranean Conference on Embedded Computing MECO - 2013 Budva, Montenegro, pp.32-35

[5] V.K. Zlobin, V.N. Ruchkin, "Neural Networks \& Neurocomputers", St.Petersburg: BHVPetersburg, 2011, $256 \mathrm{p}$.

[6] D.V. Grigorenko, V.N. Ruchkin, "Clustering of Special Data Processing Systems" in Methods and Tools for Information Processing and Storage, Ryazan: Ryazan State Radio Engineering University EPC, 2012, 118 p.

[7] V. Ruchkin, V. Romanchuk, R. Sulica, "Clustering, Restorability and Designing Of Embedded Computer Systems Based On Neuroprocessors “, $2^{\text {nd }}$ Mediterranean Conference on Embedded Computing MECO - 2013 Budva, Montenegro, pp.58-61

[8] V. Romanchuk, V. Ruchkin, "The Algorithms for the Neuroprocessor-Based Computing Structures Analysis", Вестник Рязанского государственного радиотехнического университета. 2012. № 40. С. 60-66

[9] A. Kolesenkov, B. Kostrov, V. Ruchkin, E.Ruchkina, "Antropogenic Situation Express Monitoring on The Base of Fuzzy Neural Networks", $3^{\text {rd }}$ Mediterranean Conference on Embedded Computing MECO - 2014 Budva, Montenegro, pp.166-168 
[10] T.A. Akimenko, A.A Arshakyan, E.V. Larkin, "The control over Informational Processes of the Robotc Complexes, Tula, The Tula State University Press, 2012

[11] V.S. Karpov, V.S. Ivutin, A.N. Ivutin, A.A. Suslin, "The Approach to the Realization of the Software Reliability Estimation Methodology on the Base of Complex Metric", Tula State University Herald, Technical Sciences Section, \#4, 1999, pp. 17-22.

[12] Ruchkin V.N., Romanchuk V.A., Fulin V.A. Design of the neuroprocessor systems based on the fuzzy clustering // Herald of Ryazan State Radio Engineering University. 2014. № 4(50).p. 5562 